\title{
Investigation of Transient Dispersion and Absorption Phenomena with a Bridge Type Superheterodyne Microwave Spectrometer
}

\author{
Measurement of $\mathrm{T}_{2}$ - Relaxation of $\mathrm{SO}_{2}$ and $\mathrm{OCS}$ \\ H. Bomsdorf, H. Dreizler, and H. Mäder \\ Abteilung Chemische Physik im Institut für Physikalische Chemie der Universität Kiel
}

Z. Naturforsch. 35a, 723-730 (1980); received May 19, 1980

\begin{abstract}
A bridge type superhet spectrometer for time resolved investigation of rotational relaxation is described. Allowing the application of both Stark-and pump-modulation techniques the arrangement may be used for single and double resonance experiments, showing a lot of advantages compared with a conventional Stark- or double resonance-spectrometer. These features are discussed in detail.

By means of a MW-pulse method the following two-level experiments have been carried out. The rotational relaxation time $T_{2}$ was measured by detection of M-unresolved transient emission signals for $J=1-2$ of ${ }^{16} \mathrm{O}^{12} \mathrm{C}^{32} \mathrm{~S},{ }^{16} \mathrm{O}^{12} \mathrm{C}^{34} \mathrm{~S}$ and ${ }^{16} \mathrm{O}^{13} \mathrm{C}^{32} \mathrm{~S}$ in natural isotopic abundance and for eight transitions of $\mathrm{SO}_{2}$ up to $\mathrm{J}=43$. The signals of transient dispersion were detected and compared with those of transient absorption. Their theoretical description in terms of the two-level Bloch equations is discussed.
\end{abstract}

\section{Introduction}

Bridge type microwave spectrometers with or without superheterodyne detection have been used by some authors for the measurement of rotational spectra i.e. for investigations in the frequency domain [1]. The suppression of diode flicker noise (1/f-noise) achieved with such an arrangement allows the application of small modulation frequencies and thus leads to an increase in resolution without any loss in sensitivity. For several reasons it appears to be useful to apply the same principle of operation to a spectrometer for time resolved measurements :

1. The necessity of broadband amplification in transient experiments severely restricts sensitivity. As far as $1 / f$-noise is dominant i.e. in the case of experiments using small modulation frequencies or repetition rates (below $1 \mathrm{kHz}$ ) an improvement in signal-to-noise ratio can be achieved.

2. As a result of the superhet detection principle the spectrometer is sensitive to dispersion signals. The investigation of transient dispersion is thus possible in addition to the measurement of transient absorption that can be carried out with conventional spectrometers too.

Reprint requests to Prof. Dr. H. Dreizler, Abt. Chemische Physik im Institut für Physikalische Chemie Universität Kiel, Olshausenstr. 40/60, D-2300 Kiel.
3. The MW-bridge allows the application of a certain signal source modulation method which is described in detail in section III. The transient molecular signals caused by a pulsed microwave radiation may be detected in both periods of the experiment i.e. in the on- and off-period of the signal microwave. Using a conventional spectrometer such observations can only be made by means of the Stark-switching method [2].

4. The signal power may be adjusted to the needs of the experiment without interfering with the detection sensitivity. The conversion loss of the mixer diodes is determined by the local oscillator power.

Apart from the high expense there is one main problem that restricts the use of bridge type arrangements in the frequency domain: sufficient carrier suppression can always be achieved only for a narrow frequency region. Any frequency sweep of the signal source makes involved readjustments necessary. Due to the fact that transient experiments are usually carried out with fixed MW-frequencies there are no such problems in the case of time resolved measurements.

In the present paper we first report on the features of our experimental setup which may be used for single as well as for multiple resonance transient 
experiments*. Furthermore we give the results of some two-level experiments carried out with the spectrometer: The $T_{2}$-relaxation time was measured for the $J=1-2$ transition of different isotopic species of OSC in natural abundance and for

* It is interesting to note that a similar arrangement was used by Unland and Flygare [3] for one of the first investigations of transient phenomena. Having no basic theory at their disposal and being unable to separate between absorption and dispersion signals with their apparatus the authors could not really take advantage of the superhet detection principle. eight rotational transitions of $\mathrm{SO}_{2}$. The results were obtained by means of the MW-pulse method mentioned above. Using the same modulation technique signals of transient dispersion were detected and compared with those of transient absorption. Their theoretical interpretation is briefly discussed.

\section{Experimental Setup}

A schematic block-diagram of the experimental arrangement is shown in Figure 1. The spectro-

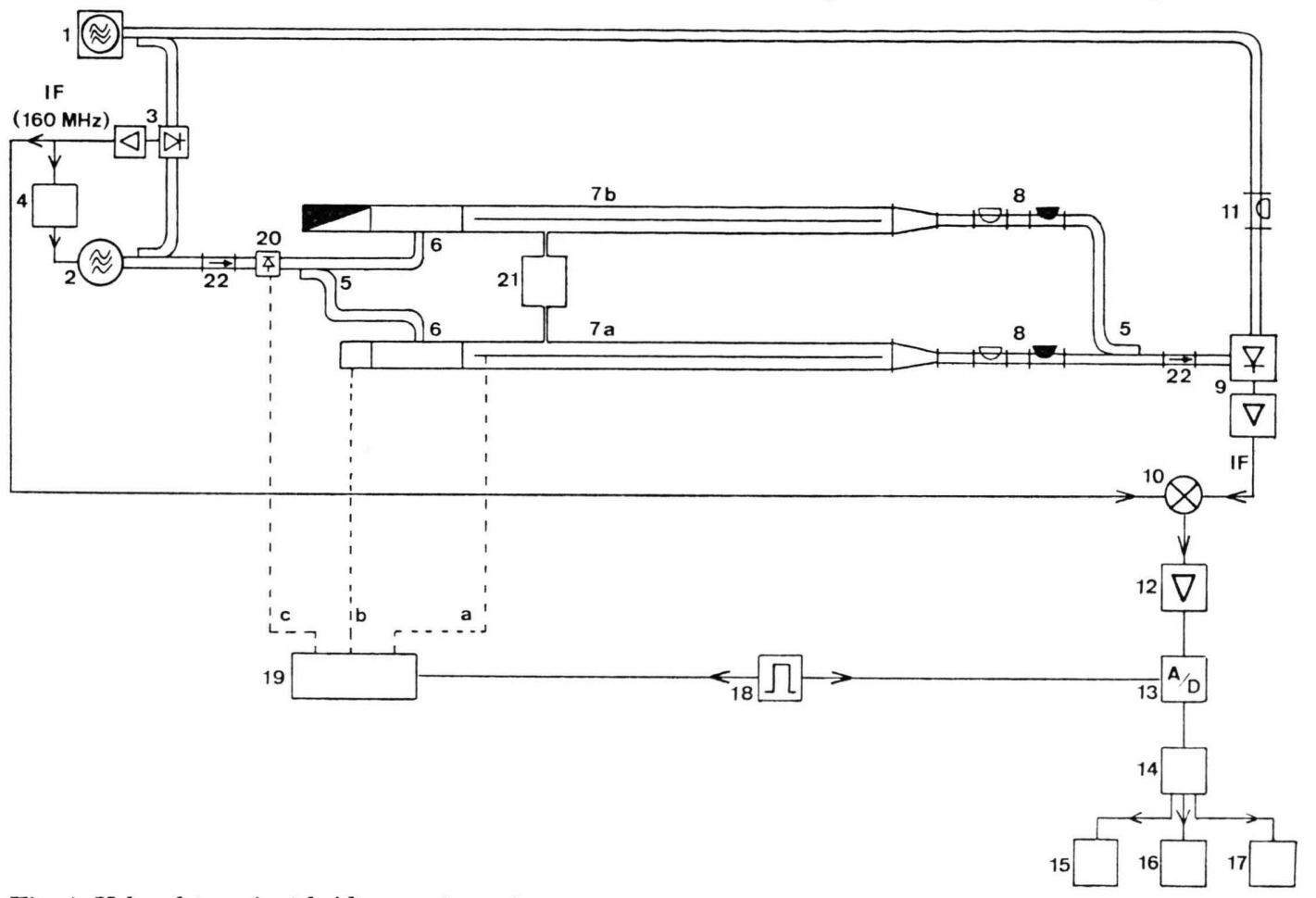

Fig. 1. K-band transient bridge spectrometer.

1. Phase stabilized local oscillator: BWO(Hp-Sweeper $8696 \mathrm{~A}), \mathrm{K}$-band $(18-26.5 \mathrm{GHz})$.

2. Signal oscillator: Klystron: OKI $24 \mathrm{~V} 11(22-26 \mathrm{GHz})$.

3. MW-mixer (double balanced) RHG; DM 18-1 B with IF-amplifier.

4. Phase stabilisation of the signal oscillator including $130 \mathrm{MHz}$ quartz [16].

5. -8. MW-bridge: (carrier suppression $>40 \mathrm{~dB}$ over a bandwidth of $\sim 1 \mathrm{MHz}$ ).

5. Directional couplers, K-band: $\mathrm{Hp} \mathrm{K} 752 \mathrm{~A}, 3 \mathrm{~dB}$.

6. Directional couplers, $\sim 10 \mathrm{~dB}$ for X-band pump MW [4].

7. Sample (7a) and reference (7 b) cell, X-band, length: $4.7 \mathrm{~m}$, including Stark septum.

8. Phase shifters and attenuators.

9. Balanced mixer with integrated broadband IF-preamplifier: Spacekom FK-U.

10. IF-mixer (double balanced): Mini Circuits ZAD-3. 11. Phase shifter.

12. Broadband amplifier: PAR mod. 115, DC-70 MHz. 13 . Fast ADC: Biomation 6500.

14. Digital averager: Fabri-TeK 1072.

16. X-Y-recorder. 17. Oscilloscope. 18. Pulse generator.

19. Modulation unit depending on experiment: a) Stark generator,

b) Pin modulated pump MW, c) Pin modulation of the signal MW.

20. Pin switch: Arra H8753 - 80 D.

21. Vacuum system including $20 \mathrm{l}$ bulb and capacitance manometer: MKS-Baratron $310 \mathrm{~B}$.

22. Unilines. Depending on the molecular signals band pass filters (between 9 and 10) and low pass filters (between 10 and 12) are inserted. 
meter has been designed for K-band signal frequencies. The signal microwave radiation is provided by a Klystron (2)** (22 to $26 \mathrm{GHz}$ ) oscillating with a fixed frequency that differs by $160 \mathrm{MHz}$ from that of a phase stabilized BWO used as local oscillator. The stabilisation (4) of the Klystron frequency is achieved by converting the $160 \mathrm{MHz}$ intermediate frequency (IF) down to $30 \mathrm{MHz}$ and using a $30 \mathrm{MHz}$ phase synchronizer. For the frequency conversion a $130 \mathrm{MHz}$-quartz oscillator in connection with a $\mathrm{RF}$ double balanced mixer is used.

The MW-bridge consists of the two X-band waveguide Stark-cells (7), attenuators and phase shifters (8) for adjustment and two $3 \mathrm{~dB} \mathrm{~K}$-band directional couplers (5). Thus it differs in construction from the commonly used "magic tee"-configuration which was found out to have a smaller bandwidth of carrier suppression.

Modulation of the molecular signal may be obtained by means of Stark-voltage or, as pump modulation for DR-experiments, using a special type of directional coupler (6) [4] to feed in X-band pump radiation. Furthermore the Stark-septum is constructed as a stripline [5] allowing pump microwaves up to a frequency of $18 \mathrm{GHz}$ to be fed into the cell through the Stark-voltage connectors. The source amplitude modulation technique already mentioned above, using the pin switch (20) in front of the bridge, is described in Section III.

The molecular signal which is the only microwave signal radiation that reaches the MW-mixer (9) after (ideal) carrier suppression by the bridge is frequency converted in two steps, first to IF, mixing with the MW-local oscillator, and then, after amplification, to DC by means of the IF-local oscillator which is provided by the stabilisation circuit. After further amplification the signals are $A / D$-converted and fed into a digital averager. Its memory contents may be displayed on an oscilloscope or an XY-recorder and can also be transferred to a PDP 11 computer.

The molecular signals of transient absorption and transient dispersion may be considered as due to electromagnetic radiation fields generated by the molecules and showing a phase difference of $\pi / 2$ (see Section III). Since the IF-mixer works as a phase sensitive detector (frequency conversion to

** Numbers refer to Figure 1.
DC) the two signals may be detected separately by choosing the relative phase of the IF-local-and the IF-signal-radiation. Thus the sensitivity of the spectrometer may be adjusted to "absorption mode" or "dispersion mode" by means of a phase shifter (11) acting on the MW-local oscillator radiation. For selecting this mode of operation one of the attenuators within the bridge is chosen to be a rotary vane type, thus showing a minimum phase change over the full attenuation range. Thereby it is possible to simulate a pure absorption signal as a standard for adjustment.

For the investigation of pressure dependence of the transient signals the cells are connected to a capacitance manometer. A $20 \mathrm{l}$ bulb is used for pressure stabilisation.

\section{Experimental and Results}

Transient two-level experiments are often carried out by means of the Stark-switching technique. The method allows the detection of transient absorption and transient emission signals but its application is usually restricted to a single $M$-component of the molecular transition, the one showing largest Stark shift. The investigation of other $M$-components is normally impossible due to the problem of unwanted "fast passage" signals * [6]. Another modulation technique is based on the generation of MWpulses by means of pin switches. This method allows the investigation of transient emission signals and thus the determination of $T_{2}$ as it was shown recently [8] employing the superhet detection principle. The detection of transient absorption signals, however, is impossible, for they coincide in time with the strong field of the MW-pulse which has to be prevented from reaching the MW-mixer by an additional pin switch. Otherwise an overload of the following amplifier would result.

Taking advantage of the carrier suppression provided by our bridge type arrangement, rejection of the MW-pulse can be obtained without a pin switch protecting the detector. Thus the transient signals created by the molecules in the sample cell ** can be detected in the absorption- as well as

* The use of additional static Stark-fields may help to avoid this problem in some favourable cases, but not in general. A static field applied during the transient emission period leads to incorrect results of $T_{2}$-measurement [7].

** The reference cell has to be held under vacuum in this type of experiment. 
in the emission-period of the experiment. Due to the limitation in suppression bandwidth of the bridge, however, the MW-pulse is not eliminated completely. Only the lower harmonics of its Fourier spectrum are suppressed. The remaining time resolved signal consists of two spikes at the beginning and at the end of the original MW-pulse (see Fig. 3), their amplitude being far too small to cause an overload of the IF-amplifiier***. For the detection of weak molecular signals a high amplification of the signal is necessary after conversion to DC. In order to prevent an overload of the amplifier following the IF-mixer, an IF-switch is used to reject the unwanted spike signals as shown in Figure 2. In Fig. 3 the influence of the switch is shown in a schematic representation. The location of the TTL-gate pulse depends on the experimental situation under consideration (MW-on-period or MW-off-period). Due to the frequency conversion to DC the transient emission signal in Fig. 3 appears as an exponential decay with zero beat frequency.

\section{III.1. Measurement of $T_{2}$ by Means of the $M W$. Pulse Method}

Analysing transient emission signals we determined the pressure dependence of the rotational relaxation time $T_{2}$ for M-unresolved transitions. Since no significant M-dependence of $T_{2}$ has been found so far and since such behaviour is also not expected [9], the M-degeneracy in our experiment

*** In our experiments MW-pulse powers up to $30 \mathrm{~mW}$ were used.



Fig. 2. Suppression of interfering residuals of the MW-pulse by an IF-switch.
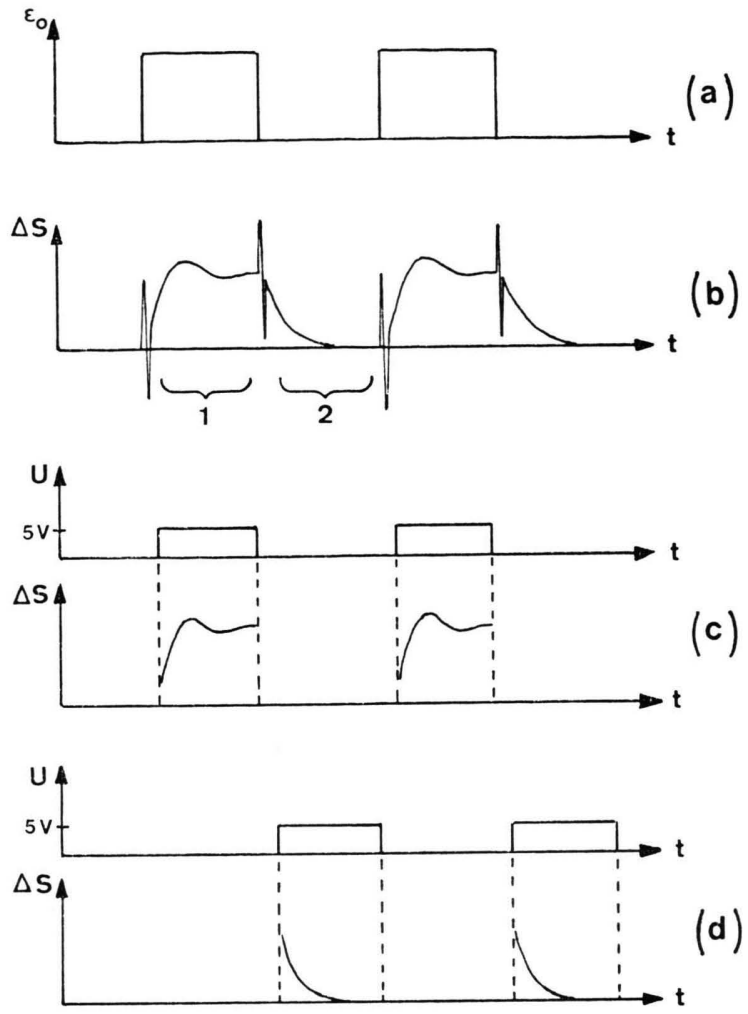

Fig. 3. Application of the IF-gate, (a) MW-pulse amplitude without carrier suppression. (b) Schematic diagram of the molecular signals and the interfering residual spikes of the MW-pulse appearing at the boundaries between the period of transient absorption (1) and transient emission (2). Resonant polarisation and good carrier suppression are assumed. (c) TTL-gate pulse for the observation of the MW-on-period and corresponding molecular signal (transient absorption). (d) same as (c) for the MWoff-period (transient emission).

should not imply a loss in information. On the other hand polarization of all M-components leads to a significant increase in sensitivity compared with the Stark-switching method. Especially the investigation of transitions with high $J$ quantum numbers (see Table 1) reported in this work would not have been possible by means of the Stark-switching technique. Furthermore, there is a technical limitation in the generation of short high voltage Stark-pulses, these being necessary for the investigation of molecular transitions showing slow Stark-effect. These problems do not arise, if the MW-pulse method is used. In order to measure $T_{2}$ with our arrangement the polarizing MW-radiation has to be exactly on resonance with the transition under investigation *.

* The transition frequencies were taken from [10] for OCS and [11] for $\mathrm{SO}_{2}$. 
Table 1. Measurement of the pressure dependence of rotational relaxation time $\mathrm{T}_{2}: 1 / \mathrm{T}_{2}=\alpha+\beta p$; OCS normal species, $\mathrm{OC}^{34} \mathrm{~S}, \mathrm{O}^{13} \mathrm{CS}$; $\mathrm{SO}_{2}$ normal species. All isotopic species were measured in natural abundance. Frequencies from [10, 11]. Temperature $300 \pm 3 \mathrm{~K}$. The given error is the double standard error of the fit.

\begin{tabular}{|c|c|c|c|c|}
\hline & Transition & Frequency $[\mathrm{MHz}]$ & $\beta\left[\mu \mathrm{sec}^{-1} \mathrm{~m}^{\text {Torr }^{-1}}\right]$ & $\alpha\left[\mu \mathrm{sec}^{-1}\right]$ \\
\hline OCS & $\mathbf{J}-\mathbf{J}^{\prime}$ & & & \\
\hline normal species & $1-2$ & 24325.930 & $0.0363 \pm 0.0004$ & $0.080 \pm 0.004$ \\
\hline $\mathrm{OC}^{34} \mathrm{~S}$ & $1-2$ & 23731.302 & $0.0346 \pm 0.0005$ & $0.093 \pm 0.006$ \\
\hline $0^{13} \mathrm{CS}$ & $1-2$ & 24247.668 & $0.0360 \pm 0.0015$ & $0.047 \pm 0.018$ \\
\hline \multirow[t]{9}{*}{$\mathrm{SO}_{2}$} & $\mathbf{J}_{\mathbf{K}-\mathbf{K}_{+}}-\mathbf{J}_{\mathbf{K}_{-}^{\prime}}^{\prime} \mathbf{K}_{+}^{\prime}$ & & & \\
\hline & $6_{1,5}-5_{2,4}$ & 23414.240 & $0.091 \pm 0.002$ & $0.068 \pm 0.010$ \\
\hline & $7_{2,6}-81,7$ & 25392.810 & $0.093 \pm 0.001$ & $0.064 \pm 0.006$ \\
\hline & $9_{1,9}-8_{2,6}$ & 24083.470 & $0.094 \pm 0.001$ & $0.059 \pm 0.006$ \\
\hline & $22_{4,18}-21_{5,17}$ & 24039.590 & $0.103 \pm 0.001$ & $0.041 \pm 0.006$ \\
\hline & $23_{5.19}-24_{4,20}$ & 22482.560 & $0.105 \pm 0.001$ & $0.060 \pm 0.006$ \\
\hline & $34_{7,27}-35_{6,30}$ & 25049.450 & $0.094 \pm 0.002$ & $0.069 \pm 0.010$ \\
\hline & $39_{8,32}-40_{7,33}$ & 23034.780 & $0.091 \pm 0.001$ & $0.056 \pm 0.008$ \\
\hline & $43_{8,36}-42_{9,33}$ & 24319.650 & $0.089 \pm 0.002$ & $0.039 \pm 0.012$ \\
\hline
\end{tabular}

Otherwise the transient emission signal does not show an exponential behaviour with zero beat frequency as assumed in our data analysis. The spectrometer sensitivity has to be adjusted to "absorption mode" since there are no dispersion phenomena in the resonance case (see text below). For the de-
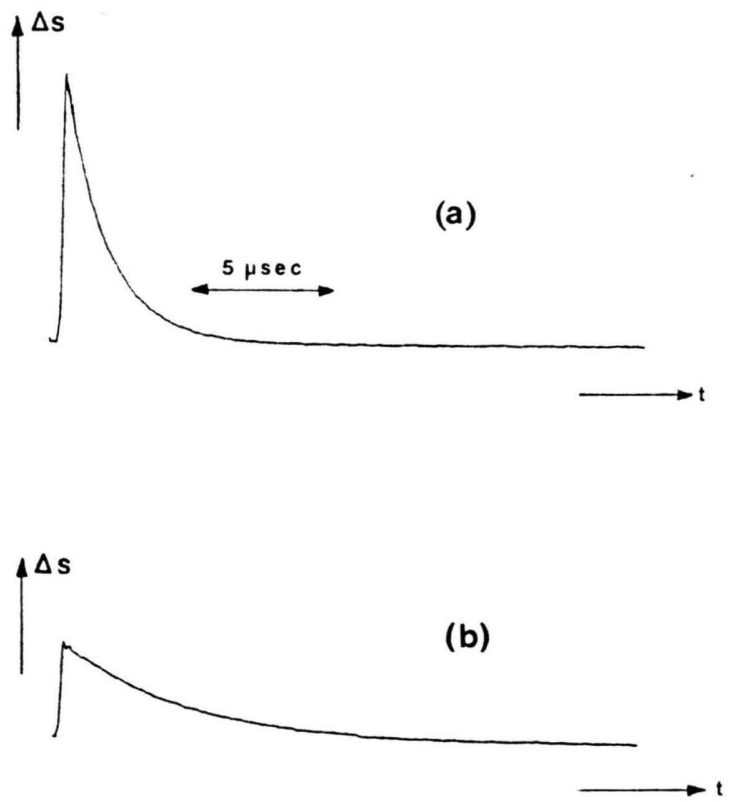

Fig. 4. Transient emission signals after resonant polarisation of $\mathrm{SO}_{2}: J_{K-} K_{+}=43_{8,36}-\mathbf{4 2}_{9,33}$ at room temperature a) $p \sim 7$ mTorr, b) $p \sim 2$ mTorr. Due to the used modulation technique (see text) an exponential decay with zero beat frequency is observed. termination of $T_{2}$ the detected signals were compared with the expression $A e^{-t / T_{2}} e^{-t^{2} / 4 q^{2}}+B$ by a least square fit program with $A, B$ and $T_{2}$ as fitting parameters. The term $e^{-t^{2} / 4 q^{2}}$ was held fixed and calculated from Doppler broadening to allow for this effect [7]. In Fig. 4 the transient emission signals of $\mathrm{SO}_{2}: J_{K_{-} K_{+}}=43_{8,36}-42_{9,33}$ are shown as an example **.

In order to obtain maximum emission amplitudes the length of the MW-pulse was chosen to give an approximate $\pi / 2$-pulse at small sample pressures $\left(t_{\pi / 2} \gtrsim 1 \mu \mathrm{sec}\right)$. A MW-power between 10 and 15 $\mathrm{mW}$ was used in the experiments. The sample pressure was varied from 1 to 25 mTorr (OCS) or 1 to $18 \mathrm{mTorr}\left(\mathrm{SO}_{2}\right)$. Slope $(\beta)$ and intercept $(\alpha)$ values of the plots of $1 / T_{2}$ against pressure are given in Table 1 . The values are averages over two (in some cases more) measurements and the given errors are twice the standard deviations. Thus they do not reflect systematic errors in our results. Due to shifts in pressure $(<0.1$ mTorr $)$ and temperature $\left(<2{ }^{\circ} \mathrm{C}\right)$, inaccuracies of the used frequency values ([10], [11]) in the $\mathrm{kHz}$-range and pin switch transient behaviour, accuracy and reproducibility of our results are restricted to about $\pm 3 \%$ of the values given in Table 1.

** To eliminate the influence of coherent pick up a baseline subtraction was carried out after each measurement. For that purpose the molecular transition frequency was switched far out of resonance with the MW-radiation by means of a static Stark-field. 
The measurement on OCS normal isotopic species was primarily carried out to check the experimental setup. The result is in good agreement with that of previous investigations [12]. No obvious difference in $T_{2}$ is observed for $\mathrm{O}^{13} \mathrm{CS}$ and $\mathrm{OC}^{34} \mathrm{~S}$ in natural isotopic abundance although there seems to be a slight deviation in the result for the latter species. Measurements on the pure isotopic substance could give further information about the relaxation mechanism.

The $T_{2}$ values found for $\mathrm{SO}_{2} \mathrm{~K}$-band transitions may be compared with those of previous measurements carried out with a MW-pulse spectrometer in the $\mathrm{Ku}$-band [8]. Our results (slopes of the plots of $1 / T_{2}$ against pressure) are consistent with these measurements showing the same dependence on $J$ quantum number: Up to a $J$ of about 20 the slope values show an increase with increasing $J$, whereas they decrease again for higher $J$ quantum numbers. In Fig. 5 this behaviour is shown considering our results as well as those of [8].

\section{III.2. Observation of Transient Absorption and Dis- persion Signals by Means of the MW-pulse Method}

The signals of transient absorption and transient dispersion arising when the MW-radiation is switched on can be detected separately as described above. In order to interpret these signals their relation to the polarisation components $P_{\mathrm{r}}$ and $P_{\mathrm{i}}$ which result from the two level Bloch equations has to be discussed. The absorption coefficient $\gamma$ of

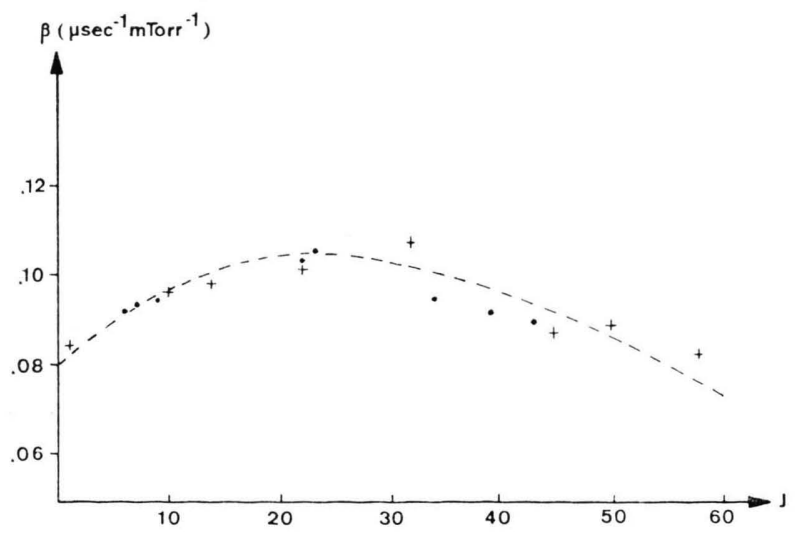

Fig. 5. Results of the $T_{2}$-measurements for different transitions of $\mathrm{SO}_{2}$. Slope values $(\beta)$ are plotted versus $J$ quantum number of the lower energy level of the transition: $\bullet$ : Measurements carried out in this work, + : results of [8]. the molecular gas sample is proportional to $P_{\mathbf{i}}$ as it is shown in [13]. A corresponding equation ean be derived for $P_{\mathrm{r}}$ and the dispersion coefficient $\delta$ as defined below.

The electric field in the absorption cell *** has to be a solution of the wave equation:

$$
\partial^{2} E_{+} / \partial z^{2}=\left(1 / c^{2}\right) \partial^{2} E_{+} / \partial t^{2}+\left(4 \pi / c^{2}\right) \partial^{2} P_{+} / \partial t^{2}
$$

with the complex polarization

$$
P_{+}=\left(P_{\mathrm{r}}+i P_{\mathrm{i}}\right) \exp \{i(\omega t-k z)\}
$$

and the expression for the field $E_{+}$:

$$
E_{+}=\varepsilon(z, t) \exp \{i(\omega t-k z)\} .
$$

$\omega$ is the frequency of the applied MW-radiation. Assuming both absorption and dispersion to be present in the sample the field amplitude must be regarded as complex too:

$$
\varepsilon(z, t)=\varepsilon_{\mathrm{r}}(z, t)+i \varepsilon_{\mathrm{i}}(z, t) .
$$

After several simplifications of the wave equation two equations for $\varepsilon_{\mathrm{r}}$ and $\varepsilon_{\mathrm{i}}$ are obtained [14]

$$
\partial \varepsilon_{\mathrm{r}} / \partial z=(2 \pi \omega / c) P_{\mathrm{i}}, \partial \varepsilon_{\mathrm{i}} / \partial z=-(2 \pi \omega / c) P_{\mathrm{r}} .
$$

Assuming a MW-radiation of amplitude $\varepsilon_{0}$ being influenced by absorption and dispersion while travelling through the sample cell, we can rewrite the complex expression for $\varepsilon(z, t)$ :

$$
\varepsilon_{\mathrm{r}}(z, t)+i \varepsilon_{\mathrm{i}}(z, t)=\varepsilon_{0} \exp \{-(\gamma(t)+i \delta(t)) z\} .
$$

Expansion of the exponential and separation of real and imaginary part gives the following expressions which are valid in the case of optically thin samples:

$$
\partial \varepsilon_{\mathrm{r}} / \partial z \approx-\gamma(t) \varepsilon_{0}, \quad \partial \varepsilon_{\mathrm{i}} / \partial z \approx-\delta(t) \varepsilon_{0} .
$$

Comparing these results with the equations above we obtain

$$
\begin{aligned}
& \gamma(t)=-(2 \pi \omega / c)\left(P_{\mathrm{i}}(t) / \varepsilon_{0}\right), \\
& \delta(t)=(2 \pi \omega / c)\left(P_{\mathrm{r}}(t) / \varepsilon_{0}\right) .
\end{aligned}
$$

Thus the observed absorption and dispersion signals may be compared with theoretical expressions for $P_{\mathrm{r}}(t)$ and $P_{\mathrm{i}}(t)$ derived from the Bloch equations.

Transient phenomena observed on OCS $J=1-2$ under different experimental conditions are shown in Figs. 6 and 7. Absorption and dispersion signals are compared. In Fig. 6 the MW-frequency is varied

\footnotetext{
*** For simplicity we assume a plane wave propagation in a lossless absorption cell.
} 

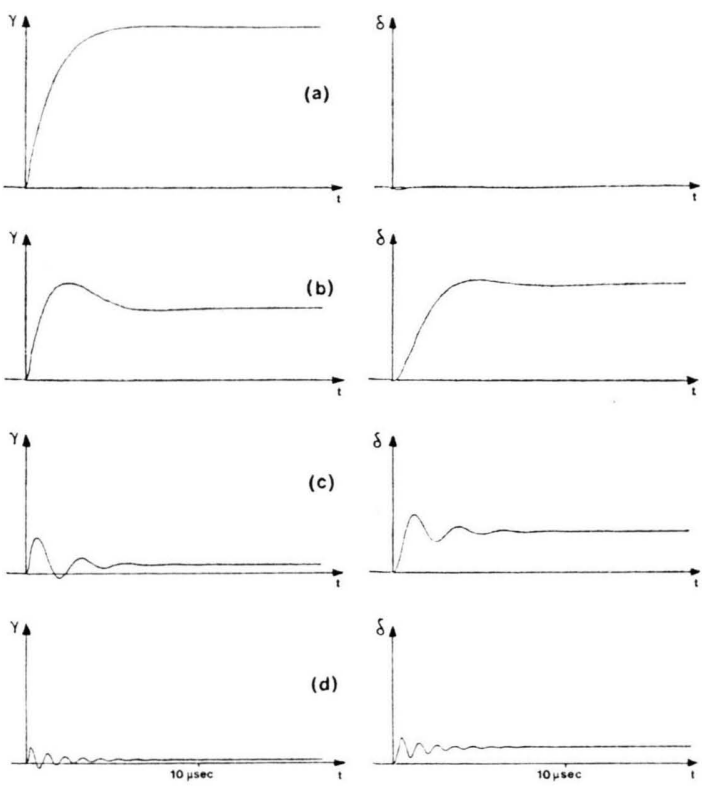

Fig. 6. Transient absorption $(\gamma)$ and dispersion $(\delta)$ signals measured on OCS: $J=1-2$ for different MW-frequencies. Pressure: $\sim 12 \mathrm{~m}$ Torr, MW-Power: $\sim 0.25 \mathrm{~mW}$. Frequency-offset $\Delta v=v_{0}-v_{\mathrm{MW}}$ :
(a) $\Delta v=0 \mathrm{kHz}$,
(b) $\Delta v=+100 \mathrm{kHz}$,
(c) $\Delta v=+400 \mathrm{kHz}$,
(d) $\Delta v=+1000 \mathrm{kHz}$.

$\gamma(t)$ and $\delta(t)$ in arbitrary units.

whereas the power $(\sim 0.25 \mathrm{~mW})$ as well as the sample pressure $(\sim 12 \mathrm{~m}$ Torr $)$ is held fixed. The frequency dependence of the steady state values reflects the shape of the unsaturated spectral line i.e. the absorption and dispersion line profiles [15] for frequencies below resonance*. Especially the much higher steady state value of $\delta$ compared to that of $\gamma$ for large frequency deviations $\Delta \omega$ becomes obvious.

The analytic solution of the Bloch equations for transient absorption has been derived in [13] using the simplifying assumption: $T_{1}=T_{2}=T$. The given result for $P_{\mathrm{i}}(t)$ representing our absorption signal is :

$$
\begin{aligned}
P_{\mathrm{i}}(t) & =\frac{\hbar \varkappa^{2} \Delta N_{0}}{4} \varepsilon_{0}\left\{e ^ { - t / T } \frac { 1 } { \Omega } \left[\left(\frac{1 / T^{2}}{\Omega^{2}+1 / T^{2}}-m_{0}\right)\right.\right. \\
& \left.\left.\cdot \sin \Omega t+\frac{\Omega \cdot 1 / T}{\Omega^{2}+1 / T^{2}} \cos \Omega t\right]-\frac{1 / T}{\Omega^{2}+1 / T^{2}}\right\}
\end{aligned}
$$

* In the case of negative frequency offset values the detected dispersion signals change sign. with

$$
\begin{aligned}
\varkappa & =2 \mu_{\mathrm{ab}} / \hbar, \quad \Delta \omega=\omega_{0}-\omega, \\
\Omega^{2} & =\varkappa^{2} \varepsilon_{0}^{2}+(\Delta \omega)^{2},
\end{aligned}
$$

and

$$
\begin{aligned}
& P_{\mathrm{r}}(0)=P_{\mathrm{i}}(0)=0, \quad \Delta N(0)=m_{0} \cdot \Delta N_{0} \\
& \left(\Delta N_{0} \text { : equilibrium value of } \Delta N\right) .
\end{aligned}
$$

In order to get the corresponding equation for $P_{\mathrm{r}}(t)$ we carried out the analogue derivation giving

$$
\begin{aligned}
P_{\mathrm{r}}(t)= & \frac{\hbar \varkappa^{2} \Delta N_{0}}{4} \varepsilon_{0}\left\{e ^ { - t / T } \frac { \Delta \omega } { \Omega ^ { 2 } } \left[m_{0}-1\right.\right. \\
& +\left(\frac{1 / T^{2}}{\Omega^{2}+1 / T^{2}}-m_{0}\right) \cos \Omega t \\
& \left.\left.-\frac{\Omega \cdot 1 / T}{\Omega^{2}+1 / T^{2}} \sin \Omega t\right]+\frac{\Delta \omega}{\Omega^{2}+1 / T^{2}}\right\} .
\end{aligned}
$$

Considering short pulses of radiation (i.e. in the case $t \ll T$ ) and making the further assumption

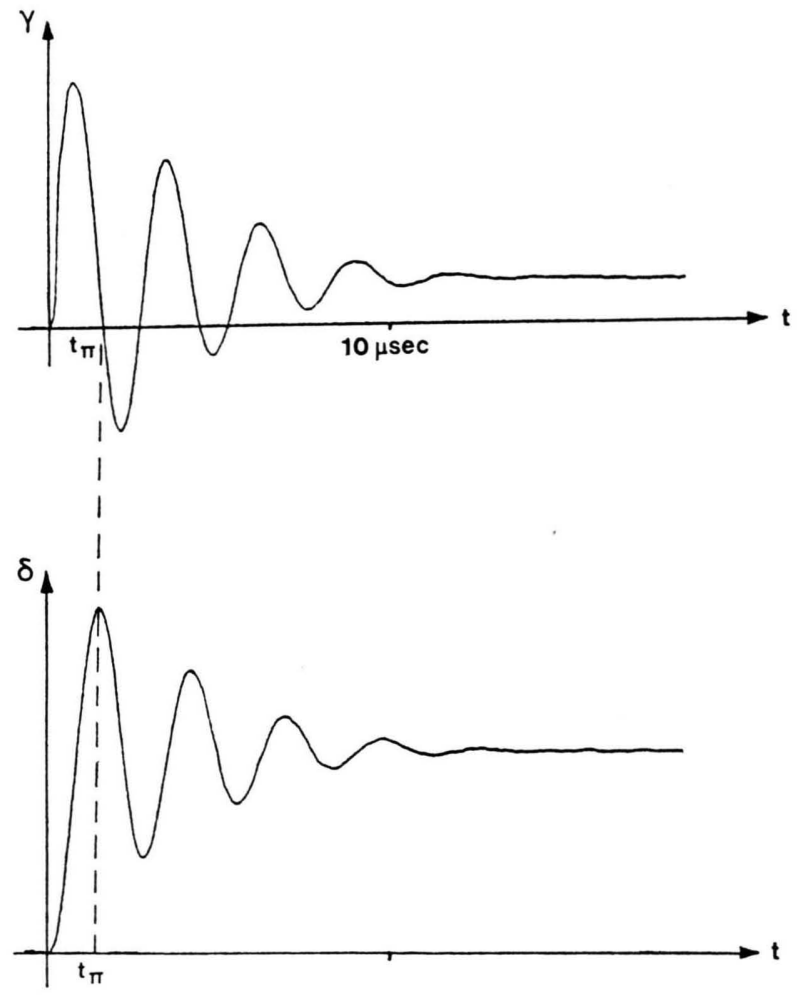

Fig. 7. Absorption- and dispersion-signals (OCS: 1-2, $\Delta v=+300 \mathrm{kHz})$ at small pressure $(\sim 1.5 \mathrm{mTorr})$ and high MW-power $(\sim 10 \mathrm{~mW})$. A pulse of length $t_{\pi}(\pi$-pulse) leads to: $\gamma\left(t_{\pi}\right)=0$ i.e. no $P_{i}$-polarisation and a maximum value of $\delta\left(t_{\pi}\right)$ i.e. maximum $P_{\text {r-polarisation. }}$ 
$\Omega \gg 1 / T$ the two equations can be reduced to

$$
\begin{aligned}
& P_{\mathrm{r}}(t)=\frac{\hbar \varkappa^{2} \varepsilon_{0} \Delta N(0)}{4 \Omega^{2}} \Delta \omega(1-\cos \Omega t), \\
& P_{\mathrm{i}}(t)=-\frac{\hbar \varkappa^{2} \varepsilon_{0} \Delta N(0)}{4 \Omega} \sin \Omega t .
\end{aligned}
$$

The concept of $\pi / 2$ - and $\pi$-pulses, that is based on these equations, is illustrated by the first few microseconds of the signals in Figure 7. Comparing the two oscillating signals, their $\pi / 2$ phase difference as well as the increased baseline of $P_{\mathrm{r}}$ becomes obvious. After a time $t_{\pi}$ (i.e. a $\pi$-pulse) the $P_{\mathrm{i}}$-signal is zero whereas there is maximum amplitude of $P_{\mathrm{r}}$. Thereby a method of producing a pure $P_{\mathrm{r}}$-polarisation is provided.

\section{Conclusion}

Employing a superhet bridge type spectrometer a number of new experimental techniques can be applied to the investigation of transient phenomena. Due to the principle of carrier suppression a pin switch method can be used for modulation of the

[1] H. D. Rudolph and D. Schwoch, Z. Angew. Phys. 31, 197 (1971), and further references in [1].

R. A. Creswell and R. H. Schwendeman, Chem. Phys. Lett. 38, 297 (1976).

[2] T. G. Schmalz and W. H. Flygare in J. I. Steinfeld (editor), "Laser and Coherence Spectroscopy" p. 134 Plenum Press. New York 1978.

[3] M. L. Unland and W. H. Flygare, J. Chem. Phys. 45, 2421 (1966)

[4] J. Ekkers, A. Bauder, and Hs. H. Günthard, Rev. Sci. Instrum. 45, 311 (1974).

[5] H. Dreizler, W. Schrepp, and R. Schwarz, Z. Naturforsch. 34a, 571 (1979).

[6] J. C. McGurk, T. G. Schmalz, and W. H. Flygare, J. Chem. Phys. 60, 4181 (1974).

[7] H. Mäder, H. Bomsdorf, and U. Andresen, Z. Naturforsch. 34a, 850 (1979).

[8] G. Bestmann, H. Dreizler, and H. Mäder, Z. Naturforsch. 34 a, 1330 (1979). signal radiation, allowing the molecular signal to be observed in both phases of the modulation period. In our experiments we took advantage of this method by using it for the determination of $T_{2}$. Because of the M-unresolved polarisation an increase in sensitivity is obtained compared with the common Stark-switch experiment. Furthermore we used the pin modulation technique for the observation of transient absorption and dispersion signals.

The sensitivity to signals of transient dispersion, or $P_{\mathrm{r}}(t)$, in addition to that of transient absorption, or $P_{\mathrm{i}}(t)$, represents the most interesting feature of our spectrometer. Thereby a second unknown of the Bloch equations becomes a measurable quantity.

\section{Acknowledgement}

We thank all members of our group for helpful discussions; Dr. H. D. Knauth for PDP11 computer facilities and the workshop of the Institute of Physical Chemistry for the workmanship in manufacture of the MW-parts. The support of the Deutsche Forschungsgemeinschaft and the Fonds der Chemischen Industrie is gratefully acknowledged

[9] W. E. Hoke, D. R. Bauer, and W. H. Flygare, J. Chem. Phys. 67, 3454 (1977).

[10] A. G. Maki, J. Phys. Chem. Ref. Data 3, 221 (1974).

[11] G. Steenbeckeliers, Ann. Soc. Sci. Bruxelles 82, 381 (1968).

[12] J. C. McGurk, H. Mäder, R. T. Hofmann, T. G. Schmalz, and W. H. Flygare, J. Chem. Phys. 61, 3759 (1974).

[13] J. C. McGurk, T. G. Schmalz, and W. H. Flygare, Adv. Chem. Phys. 25, 1 (1974).

[14] H. Mäder, Z. Naturforsch. 34a, 1170 (1979).

[15] C. H. Townes and A. L. Schawlow, Microwave Spectroscopy, p. 481 Dover Publications, Inc., New York 1975.

[16] G. Bestmann, H. Dreizler, H. Mäder, and U. Andresen, Z. Naturforsch. 35 a, 392 (1980). 